\title{
Superhydrophobic Surface by Replication of Laser Micromachined Pattern in Epoxy/Alumina Nanoparticle Composite
}

\author{
Maciej Psarski, Jacek Marczak, Jarosław Grobelny, and Grzegorz Celichowski \\ Department of Materials Technology and Chemistry, Faculty of Chemistry, University of Lodz, Pomorska 163, 90-236 Lodz, Poland \\ Correspondence should be addressed to Maciej Psarski; mpsarski@uni.lodz.pl
}

Received 17 February 2014; Accepted 12 March 2014; Published 24 April 2014

Academic Editor: Chunyi Zhi

Copyright (c) 2014 Maciej Psarski et al. This is an open access article distributed under the Creative Commons Attribution License, which permits unrestricted use, distribution, and reproduction in any medium, provided the original work is properly cited.

Superhydrophobic surfaces were obtained by superposition of microstructure-defined by replication of laser micromachined masters, with nanostructure - created by durable epoxy $/ \gamma-\mathrm{Al}_{2} \mathrm{O}_{3}$ nanoparticle composite, used for replication. Hierarchical surface topography thus obtained consisted of hexagonally spaced microcavities and nanoparticle agglomerates, exposed on the replica surface by radio frequency (RF) air plasma etching. Surface topography was further enhanced by rims around the microcavity edges, resulting from nanosecond laser micromachining defects in aluminum masters. Subsequent wet chemical hydrophobization with $1 \mathrm{H}, 1 \mathrm{H}, 2 \mathrm{H}, 2 \mathrm{H}$-perfluorotetradecyltriethoxysilane (PFTDTES) provided superhydrophobic behavior in replicas with a microcavity spacing of $30 \mu \mathrm{m}$, as indicated by a water contact angle of $160^{\circ}$ and a sliding angle of $8^{\circ}$. The preparation method is relatively simple, inexpensive, and potentially scalable.

\section{Introduction}

Superhydrophobicity is one of the most impressive examples of nature's ability to control surface wettability. Dual scale and nano- and microsized surface topography, combined with low surface free energy of the outer layer, reduce effectively to zero the attachment of water to some plant leaves (such as lotus [1]) and insect and bird wings. Superhydrophobic materials exhibit apparent water contact angles (WCA) exceeding $150^{\circ}$ and sliding angles (SA) below $10^{\circ}$ [2]. Such a wetting behavior, described by the Cassie-Baxter model [3], can inhibit accumulation of dirt, biomaterial, and in some cases ice on superhydrophobic surfaces. The phenomenon has been gaining considerable attention over the last two decades and many procedures to obtain synthetic superhydrophobic surfaces have been developed $[4,5]$, often inspired by nature $[4,6,7]$. Two general scenarios can be employed for preparation of superhydrophobic surfaces. The first one consists of the introduction of hierarchical (microand nano)roughness onto a low surface energy material (SFE below approximately $20 \mathrm{~mJ} / \mathrm{m}^{2}$ ), such as fluorinated polymers or polydimethylsiloxane (PDMS). Such materials however are rare in nature, so the second scenario employs the more ubiquitous materials with higher surface energy. The procedure here is to prepare a rough surface first and then lower its surface energy, by chemical modification. Alkyl and fluoroalkyl chloro/alkoxysilanes or fluoropolymers are the compounds commonly used for surface hydrophobization [8-11]. The techniques utilized for structure microfabrication include photolithography, nanoimprint lithography, reactive ion etching, chemical etching, soft lithography, anodization, and micro-/nanomachining [4, 5, 12-14]. Some of these techniques such as chemical etching or anodization result in stochastic topographies, whereas lithographic and micro-/nanomachining techniques can be used to generate designed, well defined structures. A range of surface geometries has been studied, encompassing pillars, grooves, hollows, and honeycombs. Wettability of such structures is usually dependent on pattern density, resulting from feature sizes and their arrangement. Closed cell structures, such as honeycomb, are particularly interesting, due to their ability to maintain superhydrophobicity under elevated water pressure, 


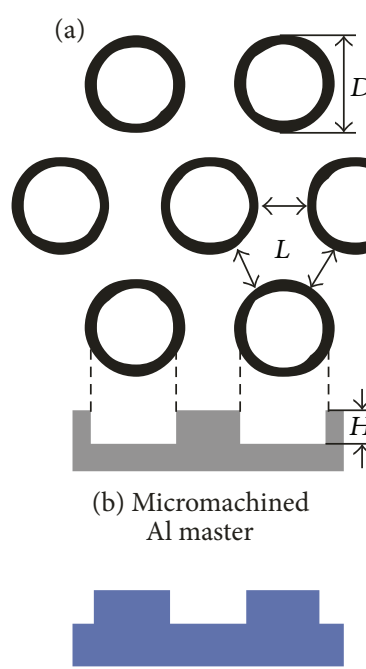

(e) Negative replica

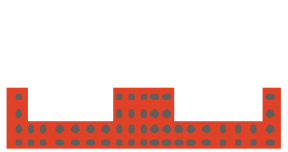

(h) Positive replica

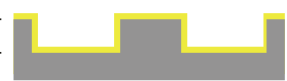

(c) Hydrophobization

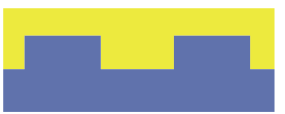

(f) Hydrophobization

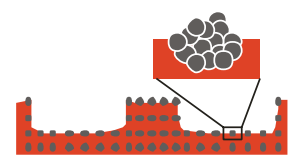

(i) RF air plasma etching

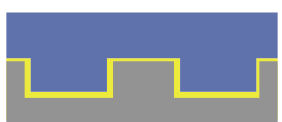

(d) PDMS casting

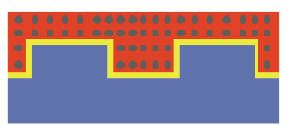

(g) Epoxy/ALNP casting

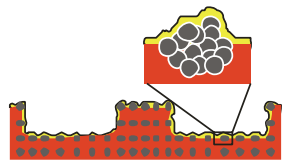

(j) Hydrophobization

$$
\begin{array}{ll}
\text { Al } & \text { ALNP } \\
- \text { PDMS } & - \text { Hydrolized PFTDTES }
\end{array}
$$

Figure 1: Schematic illustration of hierarchical surface patterning and hydrophobization. The hexagonal microcavity arrangement in the aluminum master is shown in (a), where $D=50 \mu \mathrm{m}, L=10 \mu \mathrm{m}, 30 \mu \mathrm{m}$, and $100 \mu \mathrm{m}$ for Al1, Al2, and Al3 masters, respectively. Microcavity depth $H=20 \mu \mathrm{m}$, as illustrated in the pattern cross-section (b). The consecutive replication steps consist of the master surface hydrophobization (c), casting of PDMS onto the master (d) to form a negative microstructure replica (e), hydrophobization of the PDMS replica surface (f), casting of the epoxy/ $\mathrm{Al}_{2} \mathrm{O}_{3}$ nanoparticle (ALNP) composite onto the PDMS replica (g) to obtain an epoxy nanocomposite positive replica of the master microstructure (h). The zoomed in sections illustrate the ALNP agglomerate, exposed by RF air plasma etching in (i) and surface modification with hydrolyzed PFTDTES in (j).

which translates to resistance to droplets impingement, for example, in rain conditions [15]. Such a capability is important because a transition from the nonwetting Cassie state to a fully wetting state, described by the Wenzel model [16], is the most probable material response under elevated hydrostatic pressure, resulting in pinning of droplets to the surface. Regular surface patterns can be obtained with many of the above mentioned procedures, of which nano- and micromachining provide a particularly feasible and highly reproducible means for surface texturing. Laser ablation, utilizing an ultrashort (picosecond or femtosecond) pulsed laser source, can be used for high precision machining in almost any material [17]. Femtosecond laser pulses have been utilized to produce regular arrays of nanotextured conical microstructures in metals like aluminum, copper, iron, titanium, and stainless steel [1820]. Unfortunately, the applicability of ultrashort pulsed laser texturing for a widespread use in industry is limited by high equipment costs and long machining times. Nanosecond lasers are prevailing in industrial installations instead, as a tradeoff between cost and machining quality, where the interaction of a laser beam with the workpiece often leads to defective heat-affected zone and burr formation [21, 22]. Despite these limitations, hierarchical, superhydrophobic structures on copper and steel surfaces were obtained, by a combination of microtexturing with nanosecond laser and nanotexturing by chemical etching or electrodeposition [23, 24]. Superoleophobic surfaces were obtained by overlaying nanosecond laser micropatterned Ti substrates with anodized $\mathrm{TiO}_{2}$ nanotube arrays on the top of Ti microstructure [25].

Robustness against physical damage of the intrinsically fragile superhydrophobic structures is a critical design component for real life applications and has received an increasing attention in recent years [26]. Approaches include pattern generation in hard materials, by laser micromachining or lithographic methods, in most cases combined with a secondary technique, such as plasma deposition of diamondlike coatings [27], to overlay the micropattern with durable nanostructure. Hard micro- and nanoparticles are also used to generate hierarchical roughness in polymer composite coatings $[28,29]$. We have previously reported the applicability of epoxy resin/alumina nanoparticle composites to provide the nanotexture component for the superhydrophobic structure, with elevated erosion resistance [30].

In spite of the potential of the aforementioned procedures in micro and nanostructure generation, their applications are limited by their complexity and low cost-effectiveness, 
if scaling up for industrial processes is considered [28]. Replication techniques of micro- and nanosurface geometries provide a simple and more available alternative. Soft lithography is a prominent example, including techniques such as contact printing, micromoulding, transfer printing, based on PDMS stamps, providing high quality and precision replication of micro- and nanostructures [31]. The techniques can be used to replicate natural masters, such as the lotus leaf [32], or synthetic ones. Synthetic masters can be obtained with any of the above mentioned texturing procedures, such as laser machining. A direct PDMS replica of hierarchical structures, prepared by ultrafast-laser microtexturing process, was obtained by Nayak et al. [33], exhibiting superhydrophobicity without subsequent surface treatment Park et al. [34] utilized a nickel stamp with a dual hole pattern for thermal imprinting on PDMS/carbon nanotube composite, which combined superhydrophobicity with resistance against physical damage.

The aim of this research was to propose a feasible and scalable procedure of obtaining robust superhydrophobic surfaces, by replication of regular microstructure in epoxy resin nanocomposite. The microstructure master was obtained utilizing nanosecond laser micromachining and comprised a hexagonal array of closely packed microdots in aluminum, to mimic closed cell, honeycomb photolithographic structures. Hierarchical surface roughness, necessary for superhydrophobic properties, was provided by a combination of the master micropattern with the nanostructure of the epoxy nanocomposite replica.

\section{Experimental}

Hierarchical surface topographies were prepared by replication of micropatterned masters in epoxy nanocomposite (Figure 1), followed by the enhancement of the replica surface's nanotexture and surface wet chemical hydrophobization.

2.1. Preparation of Micropatterned Masters. Micropatterned masters were obtained by laser micromachining the aluminum substrates, using a LUCE laser source $(\lambda=1064 \mathrm{~nm}$, Bright Solutions, Italy). Hexagonally spaced microcavities with diameters of approximately $50 \mu \mathrm{m}$ and depths of approximately $20 \mu \mathrm{m}$ (Figures 1(a) and 1(b)) were drilled employing a pulse repetition rate of $10 \mathrm{kHz}$ and a pulse duration between $5 \mathrm{~ns}$ and $25 \mathrm{~ns}$. Three horizontal spacings of microcavities, $L$, were chosen to investigate the effect of micropattern density on wetting properties: $L=10 \mu \mathrm{m}$ in the master labeled as All, $L=30 \mu \mathrm{m}$ in the $\mathrm{Al} 2$ master, and $L=100 \mu \mathrm{m}$ in the $\mathrm{Al} 3$ master.

2.2. Replication of Masters. Nanocomposite replicas of aluminum masters were obtained in a two-step process. First, PDMS negative replicas (Sylgard 184, Dow Corning, Midland, MI, USA, at 10:1 prepolymer to curing agent w/w proportion) were prepared from the masters. The uncured PDMS was degassed, poured onto the master (Figure 1(d)), and allowed to crosslink for 24 hours at room temperature, followed by 2 hours at $80^{\circ} \mathrm{C}$. To facilitate the removal of the

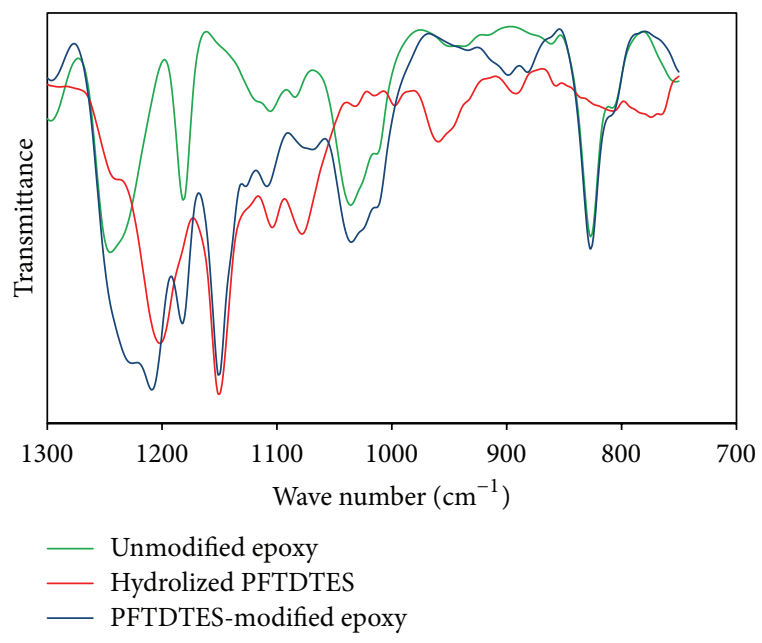

FIGURE 2: FT-IR spectra of unmodified epoxy resin, hydrolyzed PFTDTES powder, and epoxy resin after chemical modification with PFTDTES.

cured PDMS replicas from the masters, the master surfaces were hydrophobized prior to replication (Figure 1(c)), in a wet chemical process, described in Section 2.4.1.

A negative PDMS replica thus obtained served as a master in the second step of the process, for replication of the microstructure in the epoxy nanocomposite. Low viscosity diglycidyl ether of bisphenol-A epoxy resin (Epidian 5, Z. Ch. Organika-Sarzyna, Poland, the average molecular mass of 390, the epoxide equivalent weight of 192-204, the viscosity of $30000 \mathrm{mPa} \cdot \mathrm{s}$ at $25^{\circ} \mathrm{C}$ ) was mixed with aluminum oxide nanoparticles (ALNP, Nanostructured \& Amorphous Materials Inc., $\gamma-\mathrm{Al}_{2} \mathrm{O}_{3}, 99 \%$, the mean diameter of $11 \pm$ $3 \mathrm{~nm}$, as determined by atomic force microscopy [30]). Amine hardener (IDA, Z. Ch. Organika-Sarzyna) at Epidian 5/IDA weight proportion of $1: 1 \mathrm{w} / \mathrm{w}$ was then added and the mixture was ultrasonicated to be assisted with mixing. ALNP concentration of $12 \mathrm{wt} . \%$, in relation to total epoxy and hardener weight, was chosen based on our previous study [30], where such a concentration proved optimal for surface nanotexture development. Degassed nanocomposite was deposited onto PDMS negative masters (Figure $1(\mathrm{~g})$ ) and allowed to cure at room temperature for 7 days. Unfilled epoxy resin replicas were also obtained, following the same protocol as for epoxy nanocomposites, to investigate the effect of nanoparticle-derived surface nanotexture on the wetting properties of the replicas.

Similarly as in the first step, PDMS surface was modified prior to replication, to decrease nanocomposite adhesion to the negative masters (Figure 1(f)). To avoid PDMS swelling with solvent, which would occur in a wet process, gas phase modification was employed in this step and described in Section 2.4.2.

2.3. Nanotexture Exposure by RF Air Plasma Etching. After separation from PDMS, the nanocomposite surface was etched with air plasma (Zepto plasma system, Diener Electronic, Germany, $13.56 \mathrm{MHz}, 50 \mathrm{~W}$ ) to remove a thin layer 

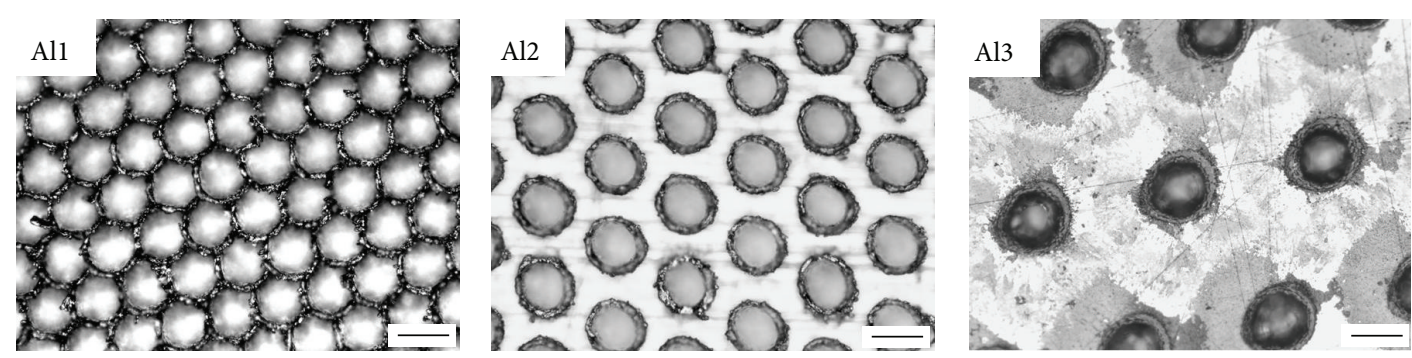

Figure 3: Optical microscopy images of aluminum masters. Microcavities horizontal spacing is $10 \mu \mathrm{m}$ (Al1), $30 \mu \mathrm{m}$ (Al2), and 100 $\mu \mathrm{m}$ (Al3). The scale bar is $50 \mu \mathrm{m}$.
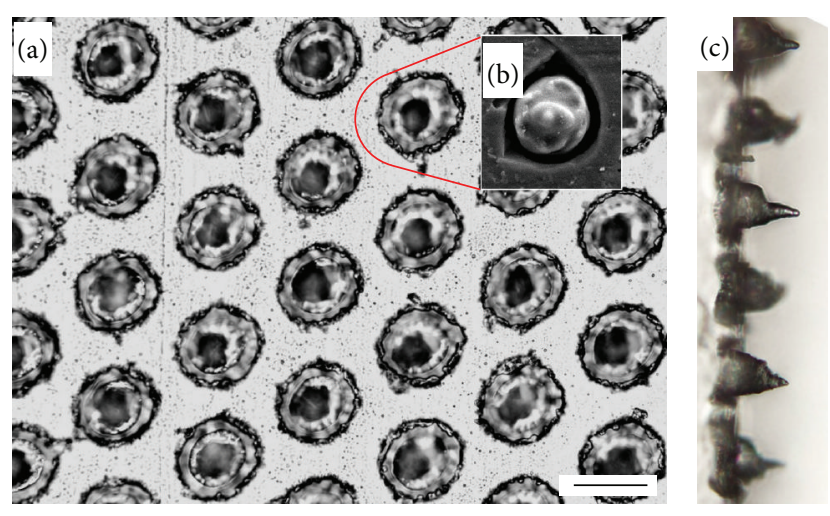

FIGURE 4: Images of PDMS negative replica-(a) optical microscopy image of AL2 master (the scale bar is $50 \mu \mathrm{m}$ ), (b) SEM detail of microcavity replica, and (c) optical microscopy image of PDMS replica cross-section, cut along the vertical direction in (a).

of the epoxy matrix and to expose the nanoparticles on the surface (Figure 1(i)). The etching time was set at 45 minutes. The process is similar to that described in [35], where silica nanoparticles were exposed on the epoxy surface by means of plasma treatment, thereby generating nanoroughness in superhydrophobic samples.

2.4. Hydrophobization of Masters and Replicas. Two surface hydrophobization procedures were employed to provide the masters with antisticking layers in the replication process and to lower the SFE of the hierarchical structures obtained to the level required for superhydrophobicity (Figure 1(j)). $\mathrm{RF}$ air plasma surface treatment (for 20 minutes at $50 \mathrm{~W}$ ) was employed before hydrophobization for the removal of impurities and generation of reactive - $\mathrm{OH}$ groups on epoxy surface [36], as well as Al-OH groups on the exposed alumina nanoparticle surface, which will readily undergo silanization [37].

2.4.1. Wet Chemical Modification. Hydrophobic thin films were obtained by dipping the specimens in a 1 vol.\% solution of $1 \mathrm{H}, 1 \mathrm{H}, 2 \mathrm{H}, 2 \mathrm{H}$-perfluorotetradecyltriethoxysilane $\left(\mathrm{CF}_{3}\left(\mathrm{CF}_{2}\right)_{11} \mathrm{CH}_{2} \mathrm{CH}_{2}-\mathrm{Si}(\mathrm{OEt})_{3}, 97 \%\right.$, ABCR $\mathrm{GmbH} \&$ Co.KG) in cyclohexane (95\%, "Chempur", Poland) solutions for $30 \mathrm{~min}$ at room temperature. After that the surfaces were rinsed with cyclohexane, dried with nitrogen gas, and heated at $80^{\circ} \mathrm{C}$ for $2 \mathrm{~h}$.

2.4.2. Modification from the Gas Phase. A 0.3 vol.\% solution of PFTDTES in hexane (95\%, "Chempur", Poland) was placed in a desiccator, along with the specimens. After evacuation of the desiccator, the deposition process was carried out in PFTDTES/hexane vapors for $2 \mathrm{~h}$. Hexane was chosen to avoid PDMS swelling that would occur in cyclohexane vapors. Then, the surfaces were removed from the desiccator and heated at $100^{\circ} \mathrm{C}$ for $2 \mathrm{~h}$. This modification was only carried out for PDMS samples, which would swell if modified in the wet process.

Mechanisms of surface silanization reactions are similar to those discussed for $1 \mathrm{H}, 1 \mathrm{H}, 2 \mathrm{H}, 2 \mathrm{H}$-perfluorodecyltrichlorosilane in our previous study [30]. In this work, a surface modifier with a longer fluorinated chain was selected for lowering the free surface energy, and the chlorosilane reactive part of compound was replaced with ethoxysilane, which is highly reactive to plasma activated nanocomposite surfaces. Substrate modification reactions were verified using FT-IR spectroscopy (a Bio-Rad FT F175 spectrometer, equipped with ATR accessory).

Two absorption maxima at 1210 and $1150 \mathrm{~cm}$, characteristic of stretching vibrations of $\mathrm{C}-\mathrm{F}$ bonds in $-\mathrm{CF}_{2}$-groups, can be recognized in the reference spectrum of PFTDTES measured for the pure hydrolyzed compound (red line in Figure 2). These bands are also present in the spectrum of PFTDTES-modified neat epoxy resin (blue line on Figure 2), which confirms the modification of the epoxy nanocomposite/resin surface (these absorption maxima are absent in the pure resin spectrum-green line in Figure 2).

2.5. Surface Characterization Methods. Surface topography of all specimens was investigated using optical microscopy (Olympus GX41 equipped with Huvitz Semiapo BF objective lenses) and scanning electron microscopy (SEM, JEOL JSM5600 , operating in secondary electron imaging mode, at accelerating voltage of $20 \mathrm{kV}$, working distance of $22 \mathrm{~mm}$ and FEI Nova NanoSEM 450, operating in secondary electron imaging mode, at accelerating voltage of $5-16 \mathrm{kV}$, working distance of $5 \mathrm{~mm}$ ). The PFTDTES deposits on the flat epoxy resin coatings were examined using atomic force microscopy (AFM, Solver P47, NT-MDT, operating in oscillation mode, in air at ambient conditions, using a MicroMasch NSC35/Al 


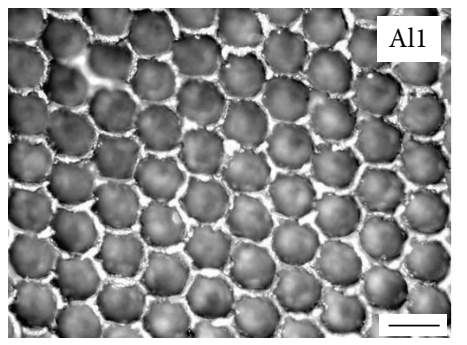

(a)

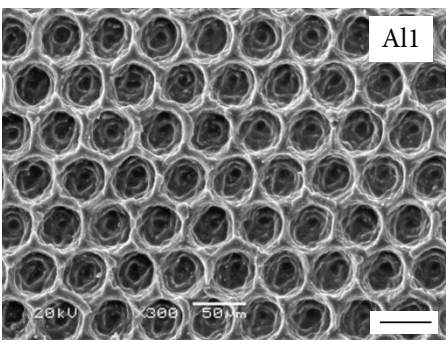

(d)

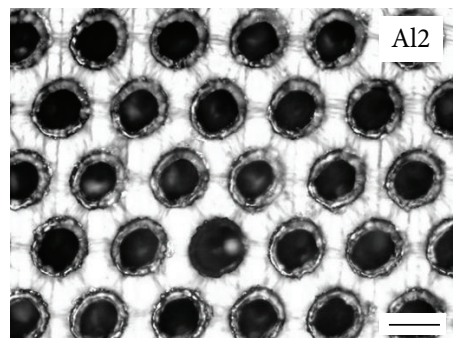

(b)

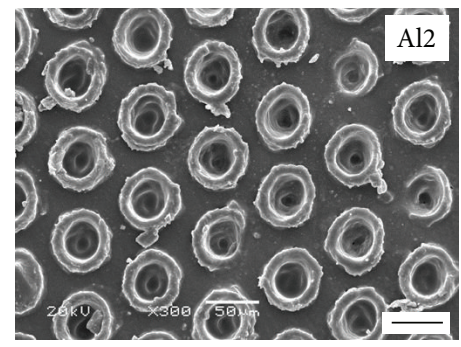

(e)

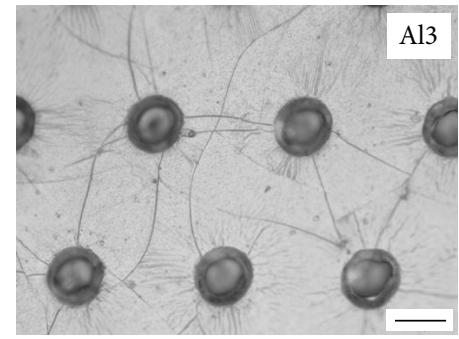

(c)

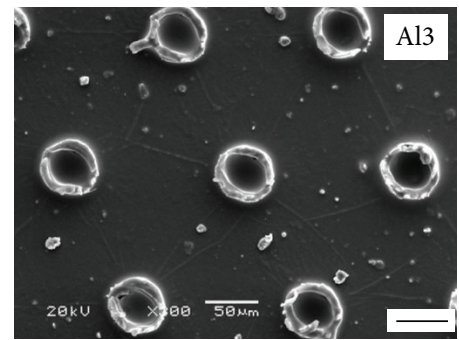

(f)

FIgURE 5: Images of Al1, Al2, and Al3 epoxy/ALNP replicas from PDMS masters-(a)-(c): optical microscopy images, (d)-(f): SEM micrographs. The scale bar is $50 \mu \mathrm{m}$.

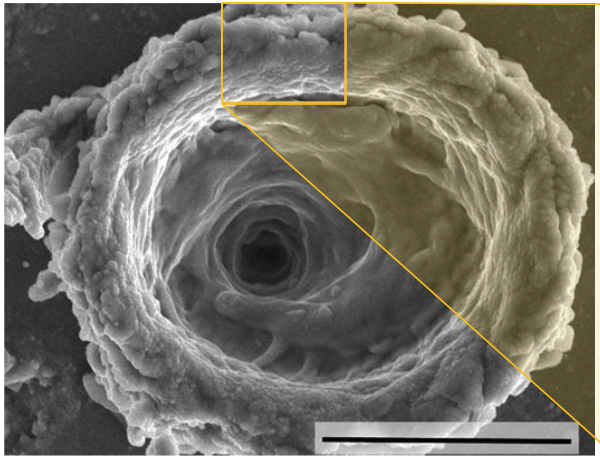

(a)

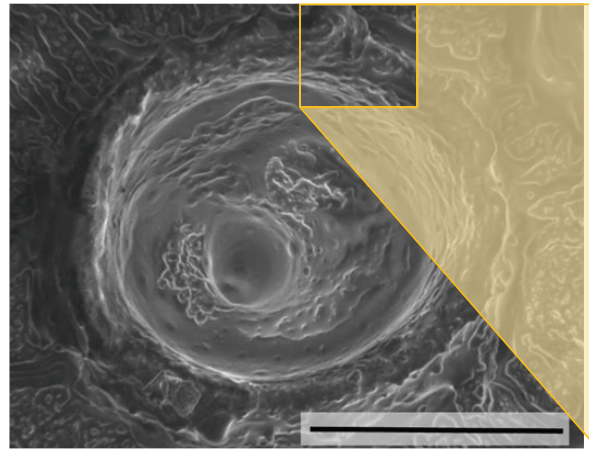

(c)

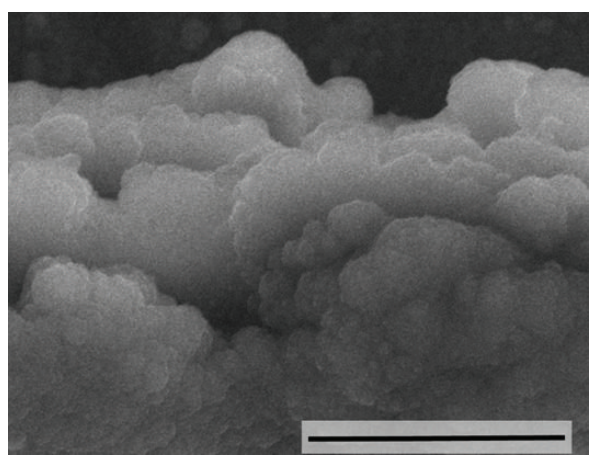

(b)

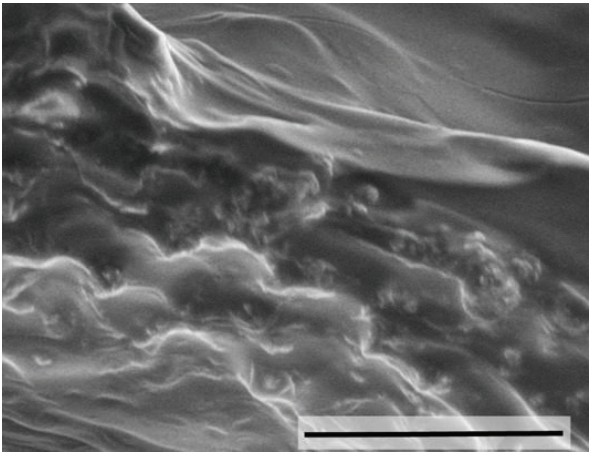

(d)

FIGURE 6: SEM micrographs of (a) laser machined microcavity in Al2 aluminum master (the scale bar is $30 \mu \mathrm{m}$ ), (b) a higher magnification image of a burr on the cavity edge (the scale bar is $5 \mu \mathrm{m}$ ), (c) microcavity in epoxy/ALNP positive replica of Al2 structure (the scale bar is $30 \mu \mathrm{m}$ ), and (d) a higher magnification image of burr on cavity edge (the scale bar is $5 \mu \mathrm{m}$ ). 
BS n-type silicon tip with radius of curvature of less than $10 \mathrm{~nm}$ and force constant of about $14 \mathrm{~N} / \mathrm{m}$, a scan size varying from $5 \times 5 \mu \mathrm{m}$ to $50 \times 50 \mu \mathrm{m}$ was used, chosen so that surface roughness did not exceed the AFM scanner's vertical range of $4 \mu \mathrm{m})$. AFM topographical images were analyzed with the Image Analysis 2 SPM image processing software (NT$\mathrm{MDT}$ ). Surface image flattening corrections employed first order line fitting (1D) and second order plane subtraction (2D).

Wettability of the surfaces was investigated using a DSA100 Drop Shape Analysis System (KRÜSS GmbH). Sessile water droplet ( $5 \mu \mathrm{L}$ of deionized water) contact angle values were measured at room temperature, by applying the LaplaceYoung fitting algorithm to the images recorded with a CCD camera. Average WCA values were obtained from measurements at five different locations on the specimen surface. Sliding angle values were determined from measurements performed on samples tilted with a motorized stage. Surface free energy values were determined from contact angle values, measured for deionized water, diiodomethane, and glycerin sessile droplets on flat surfaces, employing the van Oss-Good method.

\section{Results and Discussion}

3.1. Microtexture Replication. The aim of this work was to demonstrate a superhydrophobic surface, created by replication of a micromachined regular microtexture in a material exhibiting a nanoroughness after plasma treatment. Such a superimposition of the two textures resulted in a hierarchical surface topography, capable of superhydrophobic behavior after hydrophobization. The microtexture design consisted of hexagonally spaced cavities, as illustrated in Figure 3. The cavity diameter was defined by laser equipment characteristics (laser source wavelength, the beam projection area, pulse time, and energy). Hollows no smaller than $50 \mu \mathrm{m}$ were produced. The cavity depth of approximately $20 \mu \mathrm{m}$ resulted from the chosen machining conditions, where the beam scanned the aluminum substrate surface 10 times. Pattern density was varied and its influence on the wetting properties of the resulting nanocomposite replicas was investigated. The recast layer from the ejected molten material forms protruding irregularly shaped rims (burrs) around the cavities, which is typical for machining with long pulses [38]. Such features are normally considered as defects, but in the case of surface structuring for superhydrophobicity they proved beneficial.

PDMS negative replicas accurately reproduced the master structures, as shown in Figure 4 for the Al2 master. Cavities appear as hills while burrs are observed as indentations surrounding them (SEM micrograph inset (Figure 4(b))). A cross-section image of the PDMS replica, shown in Figure 4(c), reveals a conical shape for the cavities.

The PDMS replicas were next used as masters for replication in epoxy/ALNP composite to obtain nanocomposite positive replicas. Representative topographic images of microtextures obtained are shown in Figure 5. Accuracy of the copies is fair, if compared with original topographies from Figure 3; however, a closer examination reveals some

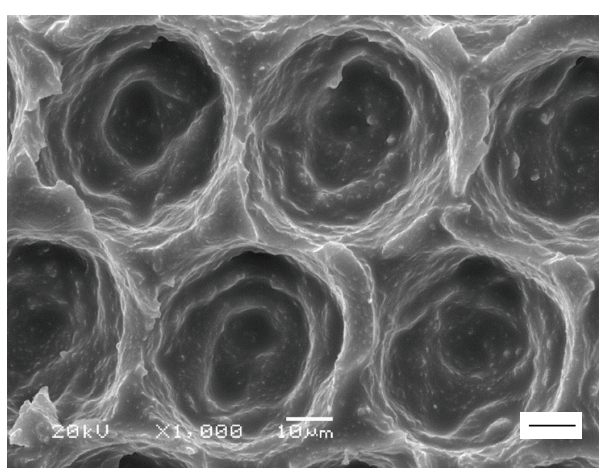

FIGURE 7: SEM image of epoxy/ALNP positive replica of All structure. The scale bar is $10 \mu \mathrm{m}$.

discrepancies at a finer length scale. Small details of the burrs in the aluminum masters (Figure 6(a)) tend to be smoothed out in epoxy/ALNP replicas (Figure 6(c)), even though they are correctly reproduced in the PDMS negative copies. The epoxy nanocomposite does not perfectly reproduce the surface microstructure. This observation may be attributed to the viscosity of the nanocomposite, which is significantly greater than that of PMDS. The replication accuracy of the All master, effectively consisting of an array of adjacent burrs, is particularly affected (Figure 7). The presence of ALNP agglomerates (Figure 6(d)) introduces nanoroughness to the surface. The contribution of nanoparticle agglomerates to surface roughness is further elaborated in Section 3.2.

3.2. Contribution of Nanoparticles to Nanoroughness. RF air plasma etching was applied to nanocomposite replicas as a means to develop surface nanoroughness. Evolution of surface nanotexture during etching is illustrated in Figure 8. Alumina nanoparticles increase the surface arithmetic mean height, $S_{a}$, from $1 \mathrm{~nm}$ for unmodified epoxy (Figure 9(a)) to $6 \mathrm{~nm}$ for untreated epoxy nanocomposite (Figure 8(a)). The epoxy matrix is gradually removed with plasma treatment time, exposing nanoparticle agglomerates on the surface. The process leads to the evolution of protrusions, or nanopillars, formed by epoxy posts, topped by ALNP agglomerates (Figure 8(b)). Nanoparticle agglomerates act as a template for epoxy anisotropic etching, similarly like in the case of colloidal lithography [39]. Formation of nanopillars is accompanied by significant evolution of surface texture in between them. Optimal development of surface nanotexture is achieved after approximately 45 minutes of plasma treatment, when densely spaced nanopillars of various heights, reaching $250 \mathrm{~nm}$ or more, are formed and $S_{a}$ reaches $27 \mathrm{~nm}$ (Figure 8(c)). Extended etching time leads to a decrease in nanopillars heights below $100 \mathrm{~nm}$ and the texture is composed of large amount of smaller protrusions, with $S_{a}$ of $7 \mathrm{~nm}$ (Figure $8(\mathrm{~d})$ ). Such topography suggests that nanopillars have been thinned by anisotropic etching and eventually collapsed. New agglomerates, at greater depths in the initial material, are also exposed, which results in the increased number of protrusions. 


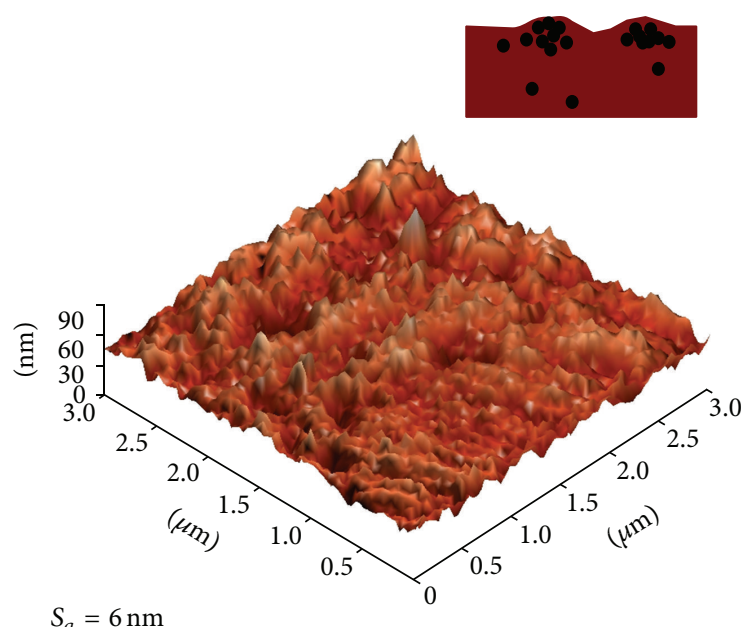

(a)

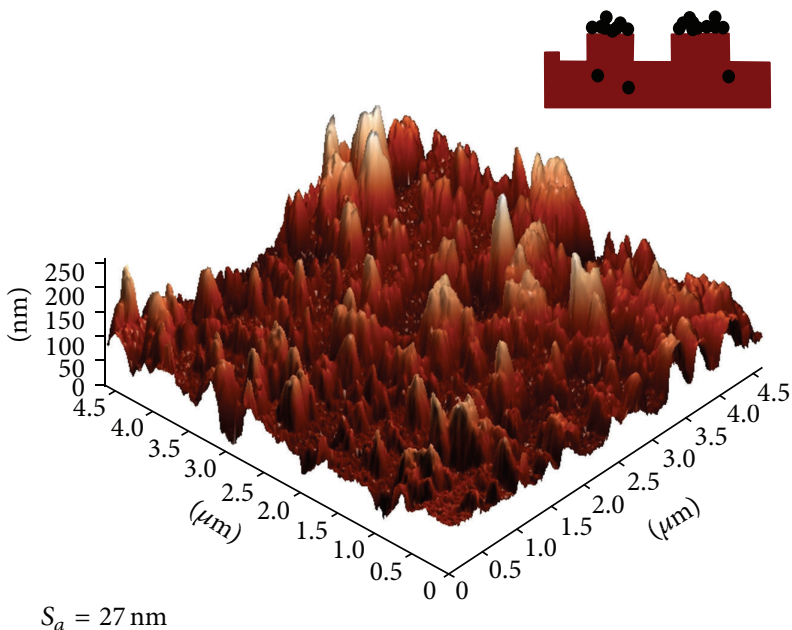

(c)

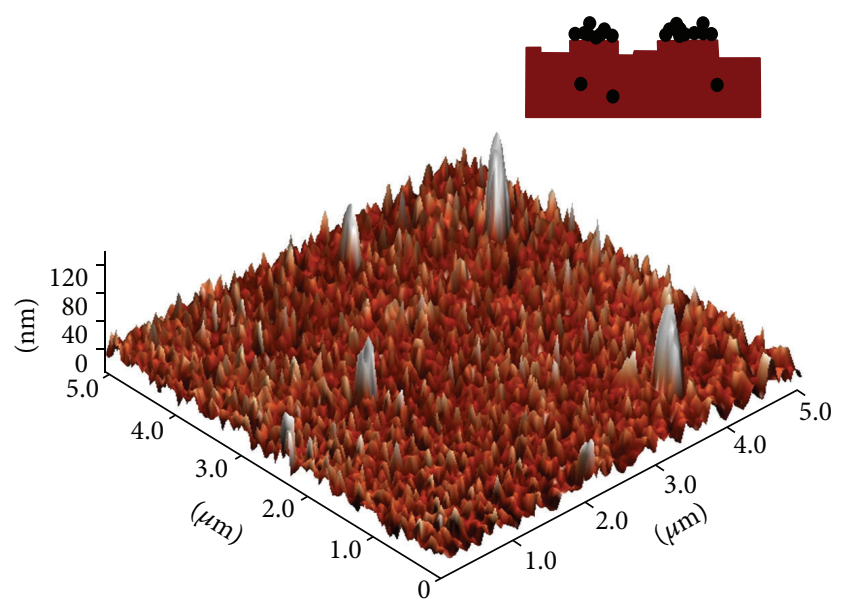

(b)

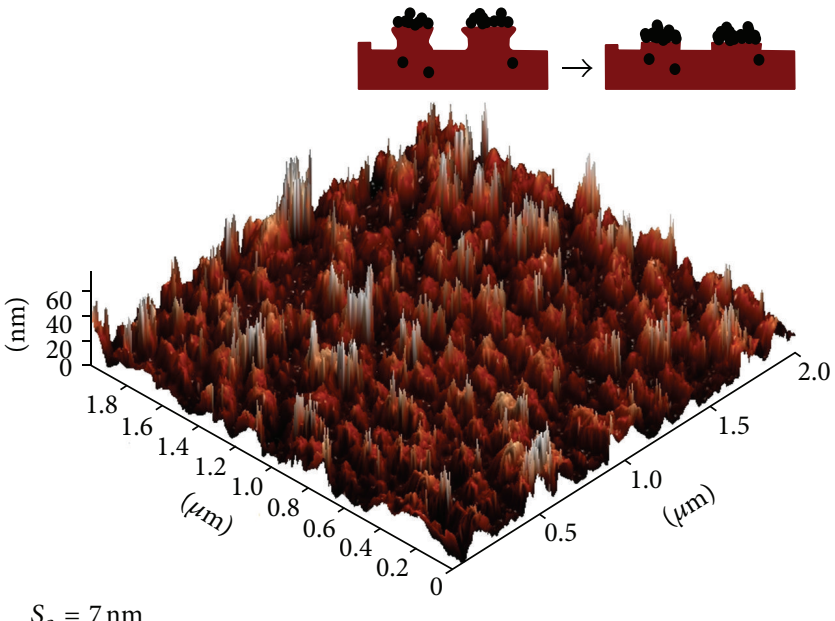

(d)

FIGURE 8: 3D visualizations of AFM topographical images, representative of epoxy/ALNP surfaces-(a) before RF air plasma etching, (b) after etching for $20 \mathrm{~min}$, (c) after etching for $45 \mathrm{~min}$, and (d) after etching for $65 \mathrm{~min}$. The insets contain schematic representations of nanostructure formation; $S_{a}$ roughness is provided for each etching stage.

3.3. Contribution of PFTDTES Deposits to Nanoroughness. Examination of the PFTDTES-modified flat epoxy resin surface topography reveals that the deposition of $1 \mathrm{H}, 1 \mathrm{H}, 2 \mathrm{H}, 2 \mathrm{H}-$ perfluorotetradecyltriethoxysilane on the surface alters the substrate roughness. Figure 9 compiles AFM topographic images, line scans, $S_{a}$ roughness, water contact angle values for surfaces of plain unmodified epoxy resin, epoxy resin after wet chemical modification with PFTDTES, epoxy/ALNP composite after air plasma etching for 45 minutes, and epoxy/ALNP composite after air plasma etching and wet chemical modification with PFTDTES.

The surface roughness of epoxy specimens increases from $1 \mathrm{~nm}$ to $6 \mathrm{~nm}$ after hydrophobization (Figures 9(a)-9(b)). The increase is caused by a build-up of nanosized PFTDTES deposit protrusions, similar to those previously reported for $1 \mathrm{H}, 1 \mathrm{H}, 2 \mathrm{H}, 2 \mathrm{H}$-perfluorodecyltrichlorosilane by Raza et al. [40] and Psarski et al. [30], where fluoroalkylsilane-derived nanoroughness contribution to surface superhydrophobicity was considered. The increase in WCA values, from $80^{\circ} \pm 1^{\circ}$ (SFE of $62 \pm 8 \mathrm{~mJ} / \mathrm{m}^{2}$ ) to $110 \pm 3^{\circ}$ (SFE of $18 \pm 2 \mathrm{~mJ} / \mathrm{m}^{2}$ ) after modification with PFTDTES, indicates hydrophobization of the epoxy surface. RF air plasma etching of epoxy/ALNP composite elevates the $S_{a}$ to $27 \mathrm{~nm}$ (Figure 9(c)), as discussed in Section 3.2. The water contact angle of $69 \pm 2^{\circ}$ recorded for these specimens indicates fully wetting Wenzel mode, typical for rough surfaces built of hydrophilic material, which is the case for the plasma treated epoxy/ALNP composite. Subsequent hydrophobization results in the contribution of PFTDTES deposits to the nanoroughness, that counterbalances some possible smoothing out of nanocomposite texture with PFTDTES film, so that the $S_{a}$ roughness is only slightly decreased to $22 \mathrm{~nm}$ (Figure 9(d)). Hydrophobic character of the specimens, resulting from partial wetting after PFTDTES treatment, is evidenced by WCA of $115 \pm 3^{\circ}$. 


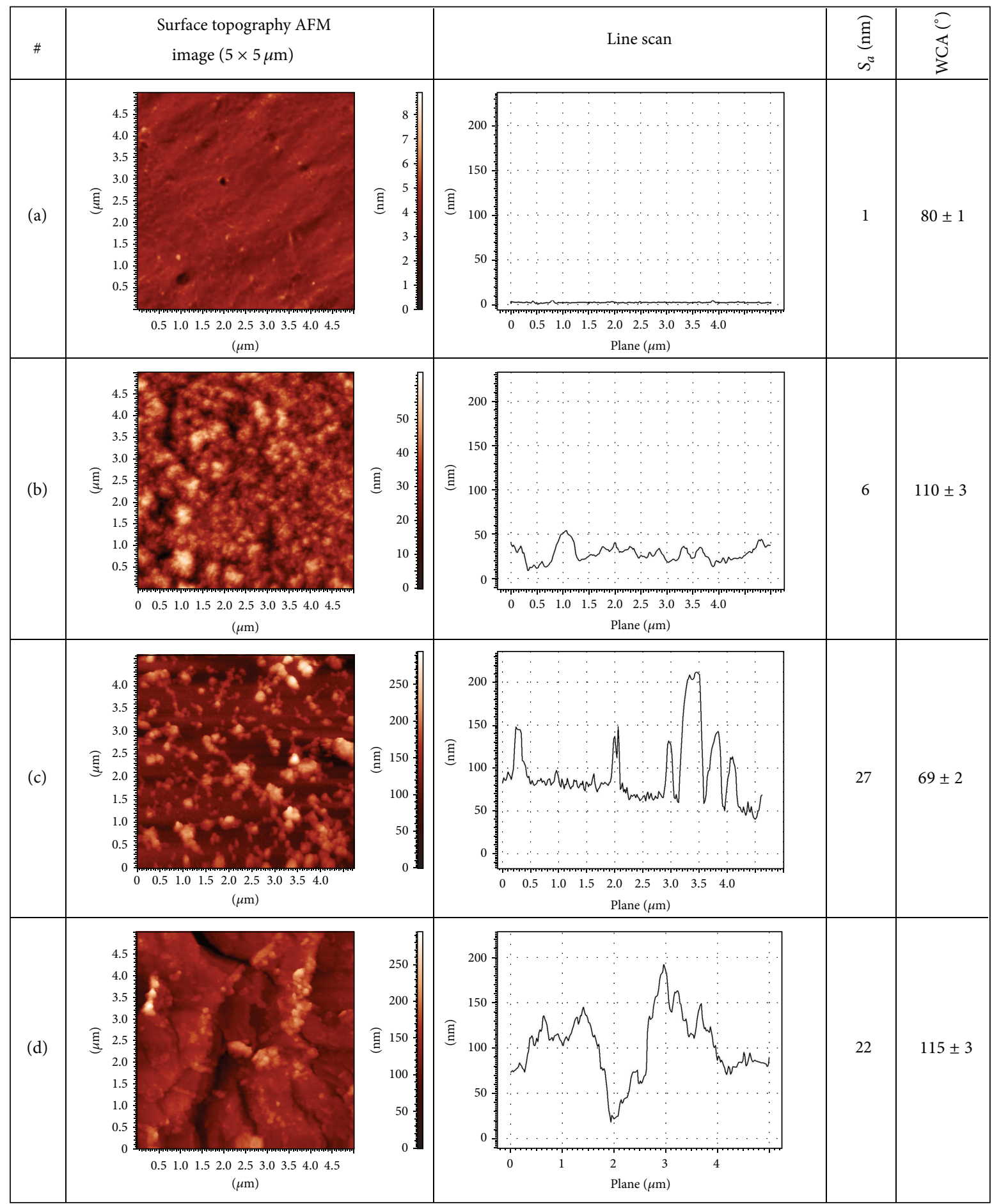

FiguRE 9: AFM surface topography images $(5 \times 5 \mu \mathrm{m})$, representative line scans, $S_{a}$ roughness, and water contact angle values for surfaces of (a) unmodified epoxy resin, (b) epoxy resin after wet chemical modification with PFTDTES, (c) epoxy/ALNP composite after air plasma etching for 45 minutes, no chemical treatment, and (d) epoxy/ALNP composite after air plasma etching and wet chemical modification with PFTDTES.

3.4. Influence of Micro- and Nanoroughness on Surface Wetting Properties. Wetting properties of surface structures were investigated during the replication procedure and are summarized in Figure 10. As-obtained aluminum masters exhibited moderate hydrophobicity (WCA in the range of
70-105 ${ }^{\circ}$ ), accompanied by strong pinning of water droplets. Liquid phase chemical modification (PFTDTES solution in cyclohexane) resulted in significant increase in water repellency of the masters, in fact Al1 and Al2 structures became superhydrophobic (WCA of $152 \pm 3^{\circ}$ and $160 \pm 2^{\circ}$, 


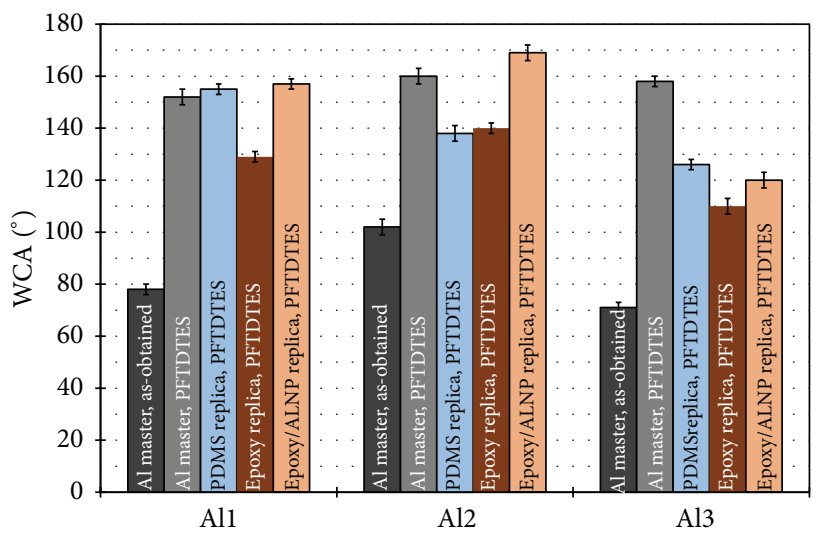

(a)

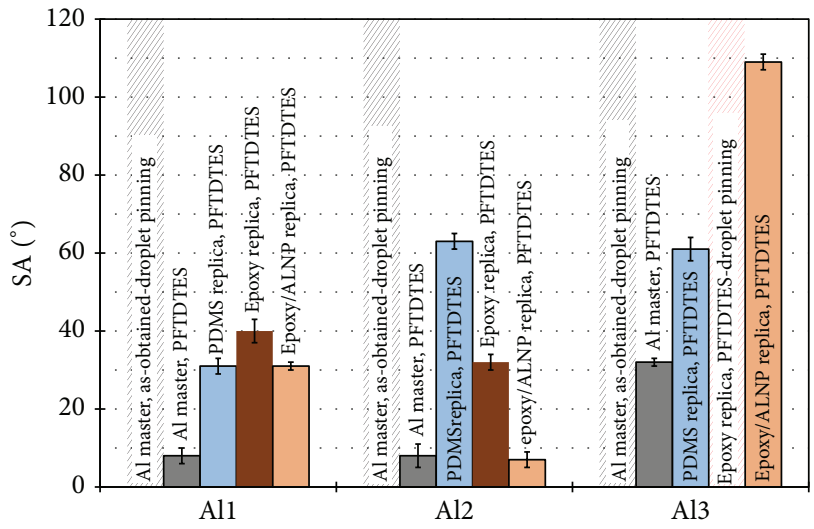

(b)

FIGURE 10: Water contact angles (a) and sliding angles (b) recorded at subsequent stages of the master replication process: unmodified Al masters, Al masters hydrophobized with PFTDTES, PDMS negative replicas hydrophobized with PFTDTES, plain epoxy positive replicas hydrophobized with PFTDTES, and epoxy/ALNP replicas hydrophobized with PFTDTES.

resp., SA of $8 \pm 1^{\circ}$ ). PDMS negative replicas exhibited nearsuperhydrophobic properties in case of All replicas after gas phase hydrophobization, hampered by considerable water droplet attachment, possibly resulting from insufficient surface nanoroughness development and full substrate wetting in the Wenzel mode (WCA of $155 \pm 2^{\circ}$ and SA of $31 \pm 2^{\circ}$ ). Reproduction of $\mathrm{Al}$ masters in plain epoxy resin, followed by liquid phase hydrophobization provided highly hydrophobic surfaces. However, as in the case of PDMS replicas, the insufficiently developed nanoscale texture component resulted in Wenzel mode wetting (WCA up to $140 \pm 2^{\circ}$, SA above $30^{\circ}$ ). Superhydrophobic behavior was attained in epoxy/ALNP composite replicas of Al2. These specimens exhibited a water contact angle of $169 \pm 2^{\circ}$ and a sliding angle of $7 \pm 2^{\circ}$.

Superhydrophobicity of $\mathrm{Al} 2$ nanocomposite replicas arises from optimal combination of several surface structure components: low surface free energy, provided by hydrophobization with PFTDTES, and hierarchical topography, composed of (a) closely packed microcavities, with horizontal spacing of $30 \mu \mathrm{m}$, surrounded by burrs and (b) nanoroughness, provided by nanoparticles exposed on the replica surface, and by PFTDTES deposits. Such a structure is sufficiently small to develop composite Cassie interface [3], required for superhydrophobicity. The contribution of burrs around the microcavities to hierarchical roughness is noteworthy, as it results from suboptimal ns laser micromachining conditions. Such surface features, normally an unwelcome byproduct of laser ablation, proved beneficial for surface hydrophobicity. Their mechanical stability in nanocomposite replicas, where they are part of a homogeneous cast, is much higher than in $\mathrm{Al}$ master, where they are formed by weakly bounded aluminum oxide recast layer. A similar "ennoblement" of process byproducts was reported by Wagterveld et al., who ascribed ultralow hysteresis of their superhydrophobic SU-8 surfaces to nanoscale debris, generated during the laser process [41]. Nanocomposite replicas of All master exhibit WCA of $157 \pm 2^{\circ}$, which is within the superhydrophobic range. However, the inaccurately reproduced fine All master structure does not provide enough water-substrate separation, resulting in droplet pinning and a high sliding angle, exceeding $30^{\circ}$. Similarly in Al3 replica, the structure does not provide sufficient support to separate the water droplet from the substrate. In this case, high water-substrate contact area and full Wenzel mode wetting of these nanocomposite replicas result due to the oversized horizontal spacing of $100 \mu \mathrm{m}$.

\section{Conclusions}

We demonstrated a feasible method of superhydrophobic surface preparation, by replication of laser micromachined hexagonal pattern of microdots in durable epoxy/alumina nanoparticle composite, followed by surface hydrophobization. Hierarchical surface structure, required for superhydrophobicity, was formed by superposition of master microtexture with nanoroughness, created by RF plasma etching of nanocomposite replica, exposing nanoparticle agglomerates on the surface. We also took advantage of nanosecond pulsed laser ablation defects, in the form of recast rims around cavities, that contributed to surface hierarchy. Inaccuracies of micropattern replication in the viscous nanocomposite were balanced out by nanoroughness formed in the plasma etching process. The effectiveness of the hierarchical structure studied depended on horizontal spacing of microcavities. Superhydrophobic behavior, exhibited by a water contact angle of $160^{\circ}$ and sliding angle of $8^{\circ}$, was observed on the replica surface when the spacing was $30 \mu \mathrm{m}$. The procedure utilizes industrially well-established techniques of ns laser ablation, replication, and RF plasma treatment, enabling the ability to scale up the processes for industrial applications.

\section{Conflict of Interests}

The authors declare that there is no conflict of interests regarding the publication of this paper. 


\section{Acknowledgments}

This work was supported by the National Science Centre of Poland through projects nos. 2011/03/N/ST8/05879 and UMO-2012/05/B/ST8/02876. The authors are grateful to Professor Weimin Liu and Professor Feng Zhou of the Lanzhou Institute of Chemical Physics, Chinese Academy of Science, for making the laser micromachining, JEOL SEM, and Drop Shape Analysis equipment available.

\section{References}

[1] W. Barthlott and C. Neinhuis, "Purity of the sacred lotus, or escape from contamination in biological surfaces," Planta, vol. 202, no. 1, pp. 1-8, 1997.

[2] P. de Gennes, D. Quere, F. Brochard-Wyart, and D. Quere, Capillarity and Wetting Phenomena, Springer, New York, NY, USA, 2004.

[3] A. B. D. Cassie and S. Baxter, "Wettability of porous surfaces," Transactions of the Faraday Society, vol. 40, pp. 546-551, 1944.

[4] B. Bhushan and Y. C. Jung, "Natural and biomimetic artificial surfaces for superhydrophobicity, self-cleaning, low adhesion, and drag reduction," Progress in Materials Science, vol. 56, no. 1, pp. 1-108, 2011.

[5] P. Roach, N. J. Shirtcliffe, and M. I. Newton, "Progess in superhydrophobic surface development," Soft Matter, vol. 4, no. 2, p. 224, 2008.

[6] K. Koch, B. Bhushan, and W. Barthlott, "Multifunctional surface structures of plants: an inspiration for biomimetics," Progress in Materials Science, vol. 54, no. 2, pp. 137-178, 2009.

[7] Z. Guo and W. Liu, "Biomimic from the superhydrophobic plant leaves in nature: binary structure and unitary structure," Plant Science, vol. 172, no. 6, pp. 1103-1112, 2007.

[8] M. Cichomski, K. Kośla, W. Kozłowski et al., "Investigation of the structure of fluoroalkylsilanes deposited on alumina surface," Applied Surface Science, vol. 258, no. 24, pp. 9849-9855, 2012.

[9] J. T. Han, Y. Jang, D. Y. Lee et al., "Fabrication of a bionic superhydrophobic metal surface by sulfur-induced morphological development," Journal of Materials Chemistry, vol. 15, no. 30, pp. 3089-3092, 2005.

[10] E. Hosono, S. Fujihara, I. Honma, and H. Zhou, "Superhydrophobic perpendicular nanopin film by the bottom-up process," Journal of the American Chemical Society, vol. 127, no. 39, pp. 13458-13459, 2005.

[11] M. Psarski, J. Marczak, G. Celichowski et al., "Hydrophobization of epoxy nanocomposite surface with $1 \mathrm{H}, 1 \mathrm{H}, 2 \mathrm{H}$, $2 \mathrm{H}$-perfluorooctyltrichlorosilane for superhydrophobic properties," Central European Journal of Physics, vol. 10, no. 5, pp. 1197-1201, 2012.

[12] Z. Guo, W. Liu, and B.-L. Su, "Superhydrophobic surfaces: from natural to biomimetic to functional," Journal of Colloid and Interface Science, vol. 353, no. 2, pp. 335-355, 2011.

[13] E. Martines, K. Seunarine, H. Morgan, N. Gadegaard, C. D. W. Wilkinson, and M. O. Riehle, "Superhydrophobicity and superhydrophilicity of regular nanopatterns," Nano Letters, vol. 5, no. 10, pp. 2097-2103, 2005.

[14] X. Liu, Q. Ye, B. Yu, Y. Liang, W. Liu, and F. Zhou, "Switching water droplet adhesion using responsive polymer brushes," Langmuir, vol. 26, no. 14, pp. 12377-12382, 2010.
[15] L. Mishchenko, B. Hatton, V. Bahadur, J. A. Taylor, T. Krupenkin, and J. Aizenberg, "Design of ice-free nanostructured surfaces based on repulsion of impacting water droplets," ACS Nano, vol. 4, no. 12, pp. 7699-7707, 2010.

[16] R. N. Wenzel, "Resistance of solid surfaces to wetting by water," Industrial and Engineering Chemistry, vol. 28, no. 8, pp. 988994, 1936.

[17] B. N. Chichkov, C. Momma, S. Nolte, F. von Alvensleben, and A. Tünnermann, "Femtosecond, picosecond and nanosecond laser ablation of solids," Applied Physics A: Materials Science and Processing, vol. 63, no. 2, pp. 109-115, 1997.

[18] B. K. Nayak and M. C. Gupta, "Self-organized micro/nano structures in metal surfaces by ultrafast laser irradiation," Optics and Lasers in Engineering, vol. 48, no. 10, pp. 940-949, 2010.

[19] A. Kietzig, S. G. Hatzikiriakos, and P. Englezos, "Patterned superhydrophobic metallic surfaces," Langmuir, vol. 25, no. 8, pp. 4821-4827, 2009.

[20] A. Kietzig, M. Negar, S. Kamal, and P. Englezos, "Laserpatterned super-hydrophobic pure metallic substrates: cassie to wenzel wetting transitions," Journal of Adhesion Science and Technology, vol. 25, pp. 2789-2809, 2011.

[21] A. Kaldos, H. J. Pieper, E. Wolf, and M. Krause, "Laser machining in die making-a modern rapid tooling process," Journal of Materials Processing Technology, vol. 155-156, no. 13, pp. 1815-1820, 2004.

[22] M. R. H. Knowles, G. Rutterford, D. Karnakis, and A. Ferguson, "Micro-machining of metals, ceramics and polymers using nanosecond lasers," International Journal of Advanced Manufacturing Technology, vol. 33, no. 1-2, pp. 95-102, 2007.

[23] C. Dong, Y. Gu, M. Zhong et al., "Fabrication of superhydrophobic $\mathrm{Cu}$ surfaces with tunable regular micro and random nanoscale structures by hybrid laser texture and chemical etching," Journal of Materials Processing Technology, vol. 211, no. 7, pp. 1234-1240, 2011.

[24] M. H. Kwon, H. S. Shin, and C. N. Chu, "Fabrication of a super-hydrophobic surface on metal using laser ablation and electrodeposition," Applied Surface Science, vol. 288, pp. 222228, 2014.

[25] D. Wang, T. Hu, L. Hu et al., "Microstructured arrays of $\mathrm{TiO}_{2}$ nanotubes for improved photo-electrocatalysis and mechanical stabili," Advanced Functional Materials, vol. 19, no. 12, pp. 19301938, 2009.

[26] C. H. Xue and J.-Z. Ma, "Long-lived superhydrophobic surfaces," Journal of Materials Chemistry A, vol. 1, no. 13, pp. 41464161, 2013.

[27] D. A. del Cerro, G. R. B. E. Römer, and A. J. Huis in’t Veld, "Erosion resistant anti-ice surfaces generated by ultra short laser pulses," Physics Procedia, vol. 5, pp. 231-235, 2010.

[28] C. H. Xue, S. T. Jia, J. Zhang, and J. Z. Ma, "Large-area fabrication of superhydrophobic surfaces for practical applications: an overview," Science and Technology of Advanced Materials, vol. 11, no. 3, Article ID 033002, 2010.

[29] D. Ebert and B. Bhushan, "Transparent, superhydrophobic, and wear-resistant coatings on glass and polymer substrates using $\mathrm{SiO}_{2}, \mathrm{ZnO}$, and ITO nanoparticles," Langmuir, vol. 28, no. 31, pp. 11391-11399, 2012.

[30] M. Psarski, G. Celichowski, J. Marczak, K. Gumowski, and G. B. Sobieraj, "Superhydrophobic dual-sized filler epoxy composite coatings," Surface and Coatings Technology, vol. 225, pp. 66-74, 2013.

[31] Y. Xia and G. M. Whitesides, "Soft lithography," Angewandte Chemie-International Edition, vol. 37, no. 5, pp. 550-575, 1998. 
[32] M. Sun, C. Luo, L. Xu et al., "Artificial lotus leaf by nanocasting," Langmuir, vol. 21, no. 19, pp. 8978-8981, 2005.

[33] B. K. Nayak, P. O. Caffrey, C. R. Speck, and M. C. Gupta, "Superhydrophobic surfaces by replication of micro/nanostructures fabricated by ultrafast-laser-microtexturing," Applied Surface Science, vol. 266, pp. 27-32, 2013.

[34] S. H. Park, E.-H. Cho, J. Sohn et al., "Design of multifunctional dual hole patterned carbon nanotube composites with superhydrophobicity and durability," Nano Research, vol. 6, no. 6, pp. 389-398, 2013.

[35] Y. Xiu, Y. Liu, B. Balu, D. W. Hess, and C. Wong, "Robust superhydrophobic surfaces prepared with epoxy resin and silica nanoparticles," IEEE Transactions on Components, Packaging and Manufacturing Technology, vol. 2, no. 3, pp. 395-401, 2012.

[36] J. A. G. Terlingen, "Functionalization of polymer surfaces," in Introduction of Functional Groups at Polymer Surfaces by Glow Discharge Techniques, Europlasma Technical Paper, chapter 2, pp. 1-29, 2004.

[37] S. H. Hyun, S. Y. Jo, and B. S. Kang, "Surface modification of $\gamma$-alumina membranes by silane coupling for $\mathrm{CO}_{2}$ separation," Journal of Membrane Science, vol. 120, no. 2, pp. 197-206, 1996.

[38] P. Bado, W. Clark, and A. Said, "Machining with long pulse lasers," http://www.cmxr.com/Education/Long.html.

[39] K. Ellinas, A. Smyrnakis, A. Malainou, A. Tserepi, and E. Gogolides, “Mesh-assisted' colloidal lithography and plasma etching: a route to large-area, uniform, ordered nano-pillar and nanopost fabrication on versatile substrates," Microelectronic Engineering, vol. 88, no. 8, pp. 2547-2551, 2011.

[40] M. A. Raza, E. S. Kooij, A. van Silfhout, and B. Poelsema, "Superhydrophobic surfaces by anomalous fluoroalkylsilane self-assembly on silica nanosphere arrays," Langmuir, vol. 26, no. 15, pp. 12962-12972, 2010.

[41] R. M. Wagterveld, C. W. J. Berendsen, S. Bouaidat, and J. Jonsmann, "Ultralow hysteresis superhydrophobic surfaces by excimer laser modification of SU-8," Langmuir, vol. 22, no. 26, pp. 10904-10908, 2006. 

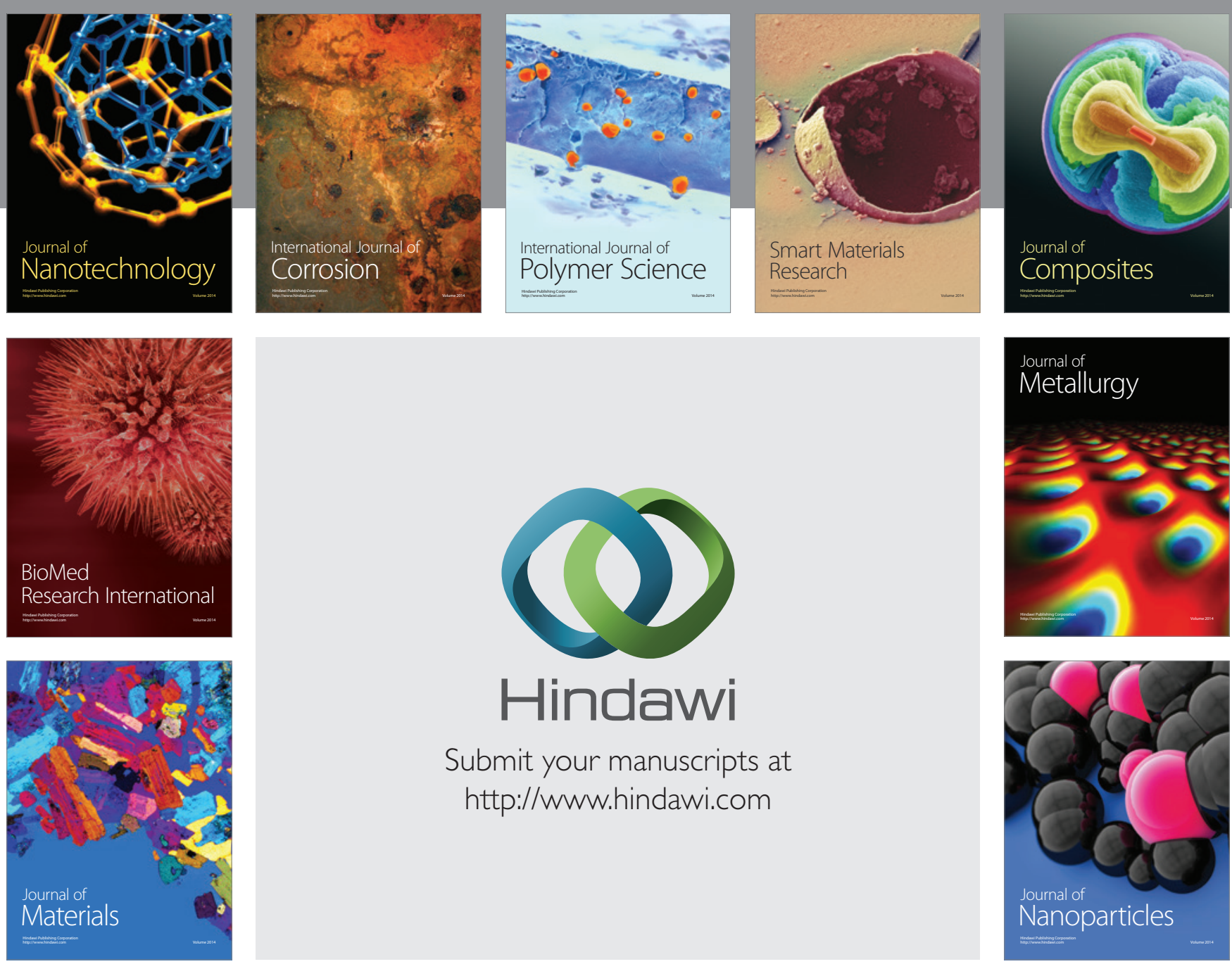

Submit your manuscripts at http://www.hindawi.com
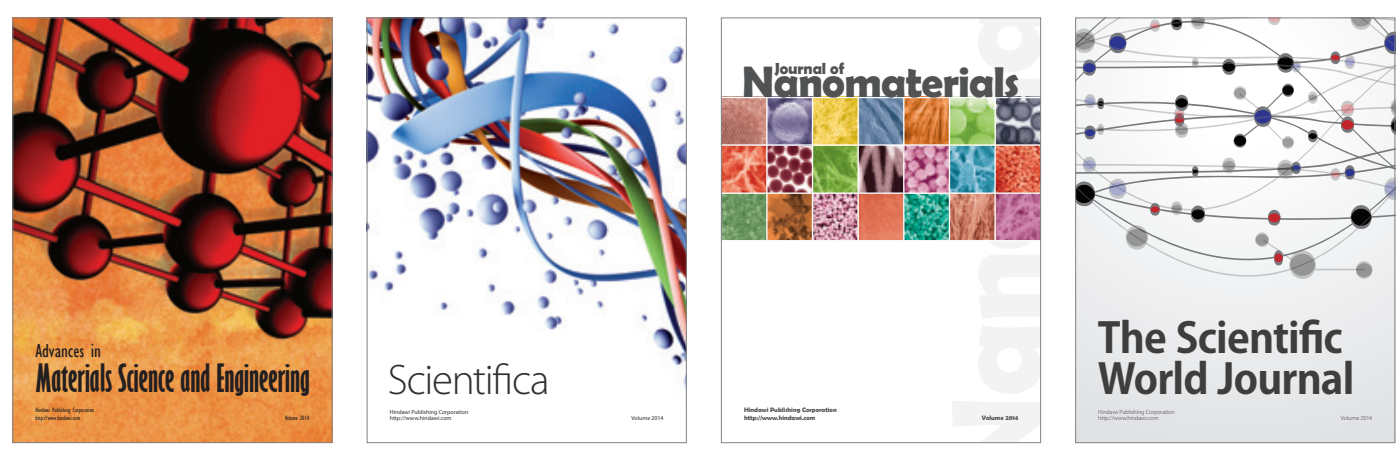

\section{The Scientific World Journal}
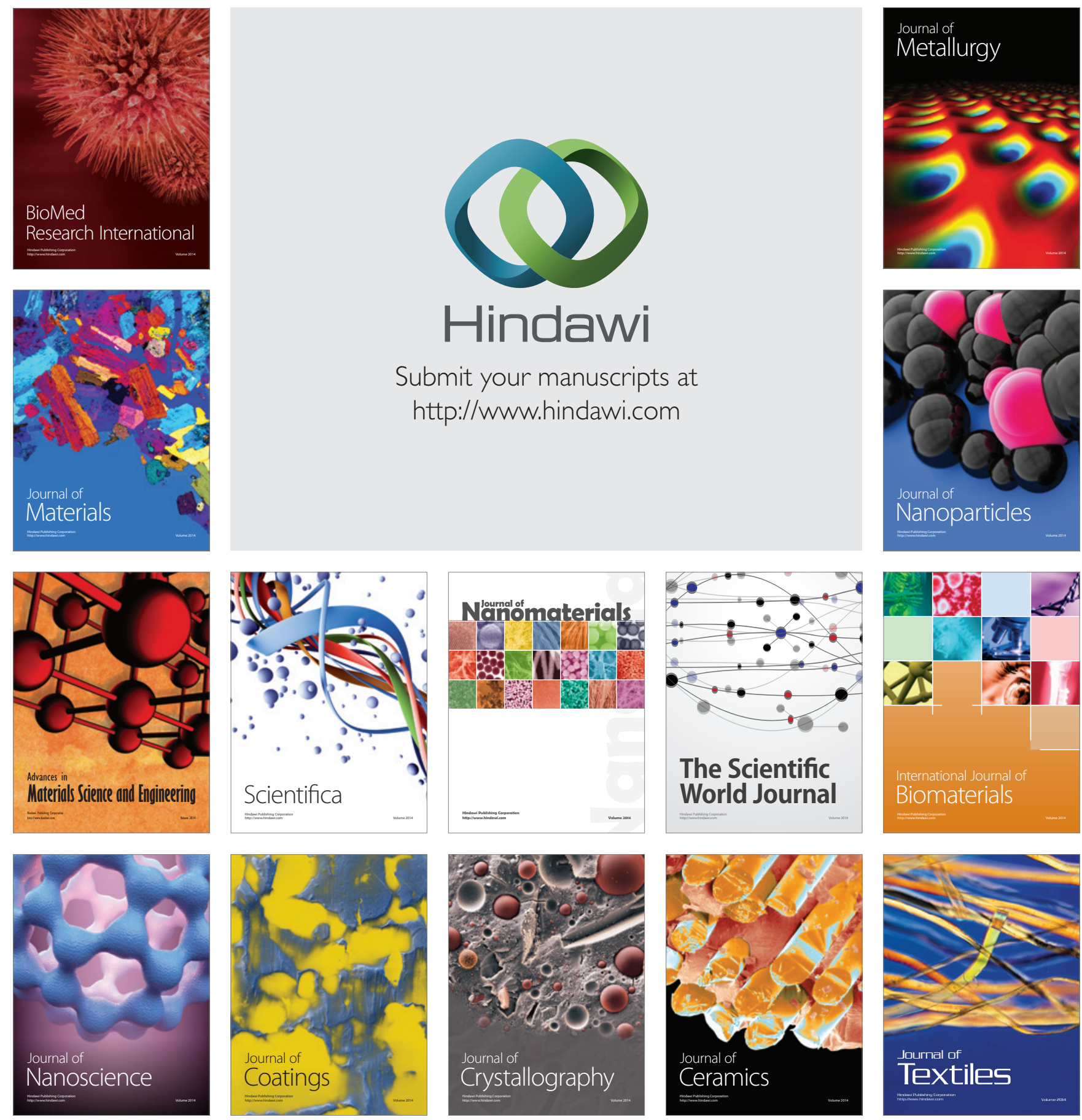